\title{
ANÁLISIS REGIONAL DE LA CONTAMINACIÓN POR PLAGUICIDAS ORGANOCLORADOS EN LECHE HUMANA EN GUERRERO, MÉXICO
}

\author{
Luis Alberto CHÁVEZ-ALMAZÁN ${ }^{1 *}$, Jesús A. DÍAZ-ORTIZ² ${ }^{2}$ Hugo A. SALDARRIAGA-NOREÑA ${ }^{3}$, \\ Gustavo DÁVILA-VÁZQUEZ $\dagger^{4}$, Agustín SANTIAGO-MORENO ${ }^{5}$, José L. ROSAS-ACEVEDO ${ }^{6}$, \\ María L. SAMPEDRO-ROSAS ${ }^{6}$, Saúl LÓPEZ-SILVA ${ }^{7}$ y Stefan M. WALISZEWSKI ${ }^{8}$
}

${ }^{1}$ Banco de Sangre Regional Zona Centro, Secretaría de Salud de Guerrero, Carretera México-Acapulco km 6+300, Campestre Santa Rosa, Chilpancingo, Guerrero, México, 39080

${ }^{2}$ Laboratorio Estatal de Salud Pública, Secretaría de Salud de Guerrero, Vicente Guerrero esq. Juan R. Escudero s/n, Renacimiento, Acapulco, Guerrero, México, 39715

${ }^{3}$ Centro de Investigaciones Químicas, Universidad Autónoma del Estado de Morelos, Universidad 1001, Chamilpa, Cuernavaca, Morelos, México, 62209

${ }^{4}$ Centro de Investigación y Asistencia en Tecnología y Diseño del Estado de Jalisco, A.C., Normalistas 800, Colinas de la Normal, Guadalajara, Jalisco, México, 44270

${ }^{5}$ Facultad de Matemáticas, Universidad Autónoma de Guerrero, Carlos E. Adame 54, Garita, Acapulco, Guerrero, México, 39350

${ }^{6}$ Centro de Ciencias de Desarrollo Regional, Universidad Autónoma de Guerrero, Los Pinos s/n, El Roble, Acapulco, Guerrero, México, 39640

${ }^{7}$ Facultad de Medicina, Universidad Autónoma de Guerrero, Solidaridad s/n, Insurgentes, Acapulco, Guerrero, México, 39300

${ }^{8}$ Instituto de Medicina Forense, Universidad Veracruzana, Juan Pablo II s/n, Costa Verde, Boca del Río, Veracruz, México, 94294

*Autor para correspondencia: chavez_79@hotmail.com

(Recibido marzo, 2017; aceptado agosto 2017)

Palabras clave: contaminantes orgánicos persistentes, exposición humana, lactancia materna

\section{RESUMEN}

Durante el siglo XX en México se aplicaron plaguicidas organoclorados intensivamente para combatir diversas problemáticas. La Campaña Nacional para la Erradicación del Paludismo implicó el uso de diclorodifeniltricloroetano (DDT), y además se utilizaron hexaclorobenceno (HCB) y $\gamma$-hexaclorociclohexano (Lindano) para la protección de semillas y cultivos agrícolas, causando una elevada exposición de la población. Por tal motivo, en este estudio se evaluó la magnitud y distribución de la contaminación por plaguicidas organoclorados en Guerrero, México, mediante el análisis de leche materna. Se recolectaron 171 muestras de madres donadoras voluntarias de las siete regiones del estado, en localidades urbanas y rurales. El análisis químico se realizó por cromatografía de gases. Las concentraciones medianas de DDT total fueron de $0.833 \mathrm{mg} / \mathrm{kg}$, mientras que el $\mathrm{HCB}$ y $\beta$-hexaclorociclohexano $(\beta-\mathrm{HCH})$ presentaron valores de 0.009 a $0.004 \mathrm{mg} / \mathrm{kg}$, respectivamente. Asimismo, se realizaron comparaciones para las concentraciones de los compuestos entre regiones. En el caso del DDT total se observaron diferencias estadísticamente significativas $(\mathrm{p}<0.05)$, siendo la Costa Chica $(0.961 \mathrm{mg} / \mathrm{kg})$ y la Tierra Caliente $(1.098 \mathrm{mg} / \mathrm{kg})$ las que mayores concentraciones presentaron. Por su parte, la Costa Grande tuvo las concentraciones de $\beta$-HCH más elevadas de todo el estado $(0.018 \mathrm{mg} / \mathrm{kg})$. Las mujeres de zonas rurales presentaron niveles superiores de 
todos los plaguicidas (con excepción del $\beta-\mathrm{HCH}$ ) que las de localidades urbanas. La información generada puede ser útil en el diseño de estudios que expliquen el papel de estos contaminantes en la salud de la población de esta región de México, y para el establecimiento de políticas públicas y programas de prevención y control.

Key words: persistent organic pollutants, human exposure, breastfeeding

\begin{abstract}
During the 20th century in Mexico, organochlorine pesticides were applied intensively to combat various problems. The National Campaign for Malaria Eradication implied the intensive use of the pesticide dichlorodiphenyltrichloroethane (DDT). On the other hand, hexachlorobenzene and $\gamma$-hexachlorocyclohexane (Lindane) were used for protection of seeds and agricultural crops, causing a prolonged exposure of population. For this reason, this study evaluated the magnitude and distribution of contamination by these organochlorine pesticides in the state of Guerrero, Mexico, through the analysis of breast milk. From both urban and rural locations, 171 samples of volunteer donor mothers were collected in the seven regions of the State. The chemical analysis was performed by gas chromatography. Median concentrations of total DDT were 0.833 $\mathrm{mg} / \mathrm{kg}$ in lipid base, while hexachlorobenzene (HCB) and $\beta$-hexachlorocyclohexane $(\beta-\mathrm{HCH})$ presented 0.009 and $0.004 \mathrm{mg} / \mathrm{kg}$, respectively. Comparisons for concentrations of the compounds between regions were made likewise. There were statistically significant differences in total DDT concentrations $(\mathrm{p}<0.05)$, with higher levels in the Costa Chica $(0.961 \mathrm{mg} / \mathrm{kg})$ and Tierra Caliente $(1.087 \mathrm{mg} / \mathrm{kg})$. Meanwhile, the Costa Grande presented the highest concentration of $\beta-\mathrm{HCH}$ throughout the state $(0.018 \mathrm{mg} /$ $\mathrm{kg}$ ). Women from rural areas had higher levels of all pesticides (with the exception of $\beta-\mathrm{HCH}$ ) than those living in urban locations. The information generated may be useful to the design of studies that explain the role of these pollutants in the health of the population of this region of Mexico, and for the establishment of public policies and prevention and control programs.
\end{abstract}

\section{INTRODUCCIÓN}

Desde mediados del siglo XX en la República Mexicana se aplicaron de manera intensiva plaguicidas organoclorados para diversos usos, con la finalidad de brindar numerosos beneficios al hombre. El diclorodifenitricloroetano, comúnmente llamado DDT, se utilizó durante la Campaña Nacional para la Erradicación del Paludismo, siendo Guerrero una de las entidades federativas con mayor riesgo de transmisión de la enfermedad; por lo tanto, ahí fueron mayores las necesidades de aplicación del insecticida para controlar el vector (GallardoDíaz et al. 2000). Entretanto, el Lindano, isómero $\gamma$-hexaclorociclohexano $(\gamma$-HCH), y el hexaclorobenceno (HCB) se emplearon en la protección de semillas y cultivos para mejorar la producción agrícola y para la eliminación de ectoparásitos en el ganado y el hombre. Estos compuestos poseen una gran estabilidad química, son de naturaleza lipofilica y tienden a acumularse en los distintos ambientes así como en los seres vivos. También se les conoce también como disruptores endocrinos por su actividad estrogénica y antiandrogénica en modelos animales y el hombre, lo que ha generado un creciente interés por investigar su papel en el desarrollo de enfermedades como resultado de la exposición crónica (de Roos et al. 2005, RaaschouNielsen et al. 2005).

Los plaguicidas organoclorados pueden monitorearse en poblaciones humanas a través del análisis de leche materna, lo que refleja en parte la cantidad de contaminante presente en el ambiente de donde fue absorbido por los individuos estudiados (IPCS 2011). Existen escasos estudios sobre estos contaminantes orgánicos persistentes en la población del estado de Guerrero (Chávez-Almazán et al. 2014, 2016). Respecto a la distribución de estos compuestos en el estado, no hay información que permita identificar las diferencias en la exposición entre sus 
habitantes y de esta forma brindar un panorama de la problemática existente que coadyuve a la toma de decisiones de las instituciones públicas ambientales y sanitarias tendientes a disminuir el contacto con estos contaminantes y prevenir sus efectos adversos a largo plazo en las personas expuestas.

El objetivo del trabajo fue conocer los niveles de plaguicidas organoclorados en muestras de leche materna madura de mujeres de diferentes regiones y municipios del estado de Guerrero, México. Además, determinar la influencia de factores como el tipo de localidad (rural y/o urbana) y región de residencia, sobre las concentraciones encontradas. Los niveles de contaminación por plaguicidas organoclorados en las diferentes regiones de Guerrero se presentan gráficamente para un análisis práctico y sencillo de la problemática relacionada con la permanencia de estos compuestos.

Una de las aportaciones de este trabajo es que la información generada puede ser de utilidad para el desarrollo de investigaciones futuras, proporcionando una base sobre el estado de la contaminación por plaguicidas organoclorados en la región para establecer posibles relaciones con el perfil epidemiológico de Guerrero.

\section{MATERIALES Y MÉTODOS}

\section{Área de estudio}

El estado de Guerrero se localiza al sur de México entre las coordenadas $16^{\circ} 18^{\prime}-18^{\circ} 48$ de latitud norte y $98^{\circ} 03^{\prime}-102^{\circ} 12^{\prime}$ de longitud oeste. Posee un clima cálido subhúmedo en la mayor parte de su territorio, la precipitación anual promedio es de $1200 \mathrm{~mm}$. Cuenta con 81 municipios y está dividido en siete regiones político administrativas, a saber: Tierra $\mathrm{Ca}-$ liente, Norte, Centro, Montaña, Costa Grande, Costa Chica y Acapulco (Fig. 1). De acuerdo con el Censo de Población y Vivienda de 2010 llevado a cabo por el Instituto Nacional de Estadística y Geografía de México (INEGI), su población consta de 3388738 hab, con 1743207 mujeres y 1645561 hombres (INEGI 2010).

\section{Diseño del estudio}

Este es un estudio de tipo prospectivo, transversal, observacional y comparativo. La población de estudio fueron mujeres en etapa de lactancia de 2 a 8 semanas, habitantes del estado de Guerrero durante al menos los últimos 10 años, las cuales fueron clasificadas de acuerdo con la región y el tipo de localidad donde residen.
Se recolectaron muestras de leche de aproximadamente 30 a $50 \mathrm{~mL}$ en frascos de vidrio con tapa de teflón previamente lavados con mezcla crómica, las cuales se transportaron al laboratorio a una temperatura de 2 a $10{ }^{\circ} \mathrm{C}$. Posteriormente se centrifugaron durante $15 \mathrm{~min}$ a $3000 \mathrm{rpm}$; las fracciones de grasa resultantes fueron transferidas a viales de vidrio y se almacenaron a $-20^{\circ} \mathrm{C}$ en un congelador biomédico (Sanyo Electric Co, Ltd, Japón) hasta su análisis.

\section{Análisis de muestras}

La técnica de análisis de residuos de plaguicidas organoclorados en grasa de leche humana y/o de origen animal fue realizada de acuerdo con la metodología descrita por Waliszewski et al. (2004), en la cual se extraen los compuestos de interés con hexano, seguido de precipitación de las grasas por adición de ácido sulfúrico concentrado, así como eliminación de residuos que pudieran causar problemas en la medición de las señales analíticas generadas.

Se colocaron de 2 a $3 \mathrm{~g}$ de grasa de leche en un mortero y se mezclaron con sulfato de sodio anhidro (Sigma-Aldrich, St. Louis, MO, USA) hasta obtener un homogenizado seco, posteriormente este material se colocó en una columna de vidrio y se dejaron pasar $100 \mathrm{~mL}$ de hexano grado HPLC (Caledon Laboratories Ltd. Georgetown, Ont. Canada) que fue recuperado en un matraz de bola y concentrado a $30 \mathrm{~mL}$ aproximadamente, en un evaporador rotatorio a $40{ }^{\circ} \mathrm{C}$. Para determinar la cantidad de grasa contenida en la muestra, se colocaron $10 \mathrm{~mL}$ del extracto en un matraz de bola previamente pesado, luego se evaporó totalmente el disolvente, y se determinó la cantidad de grasa por las diferencias de peso. En un tubo de ensayo se transfirieron $10 \mathrm{~mL}$ del extracto concentrado y se agregó $1 \mathrm{~mL}$ de ácido sulfúrico concentrado (Schwefelsaure 95-97 \% zur Analyse, Merck, Darmstadt, Germany), se agitó la mezcla vigorosamente durante 1 min y se dejó reposar unos minutos para permitir la separación de fases. El sobrenadante se filtró por una capa de aproximadamente $5 \mathrm{~g}$ de sulfato de sodio anhidro, se enjuagó con hexano y los líquidos eluidos se recuperaron en un matraz de bola, después se evaporó el disolvente y se transfirió de manera cuantitativa a un vial de vidrio a un aforo de $1 \mathrm{~mL}$ con hexano. Se inyectó $1 \mu \mathrm{L}$ de la muestra en modo "sin división" en un cromatógrafo de gases con detector de captura de electrones marca Varian, modelo 3400CX (Palo Alto, CA, USA) equipado con una columna capilar DB-608 de 0.32 $\mu \mathrm{m}$ de diámetro y $30 \mathrm{~m}$ de longitud, con un flujo de nitrógeno de $6.3 \mathrm{~mL} / \mathrm{min}$. La temperatura del horno inicialmente fue de $193{ }^{\circ} \mathrm{C}$ y permaneció constante 


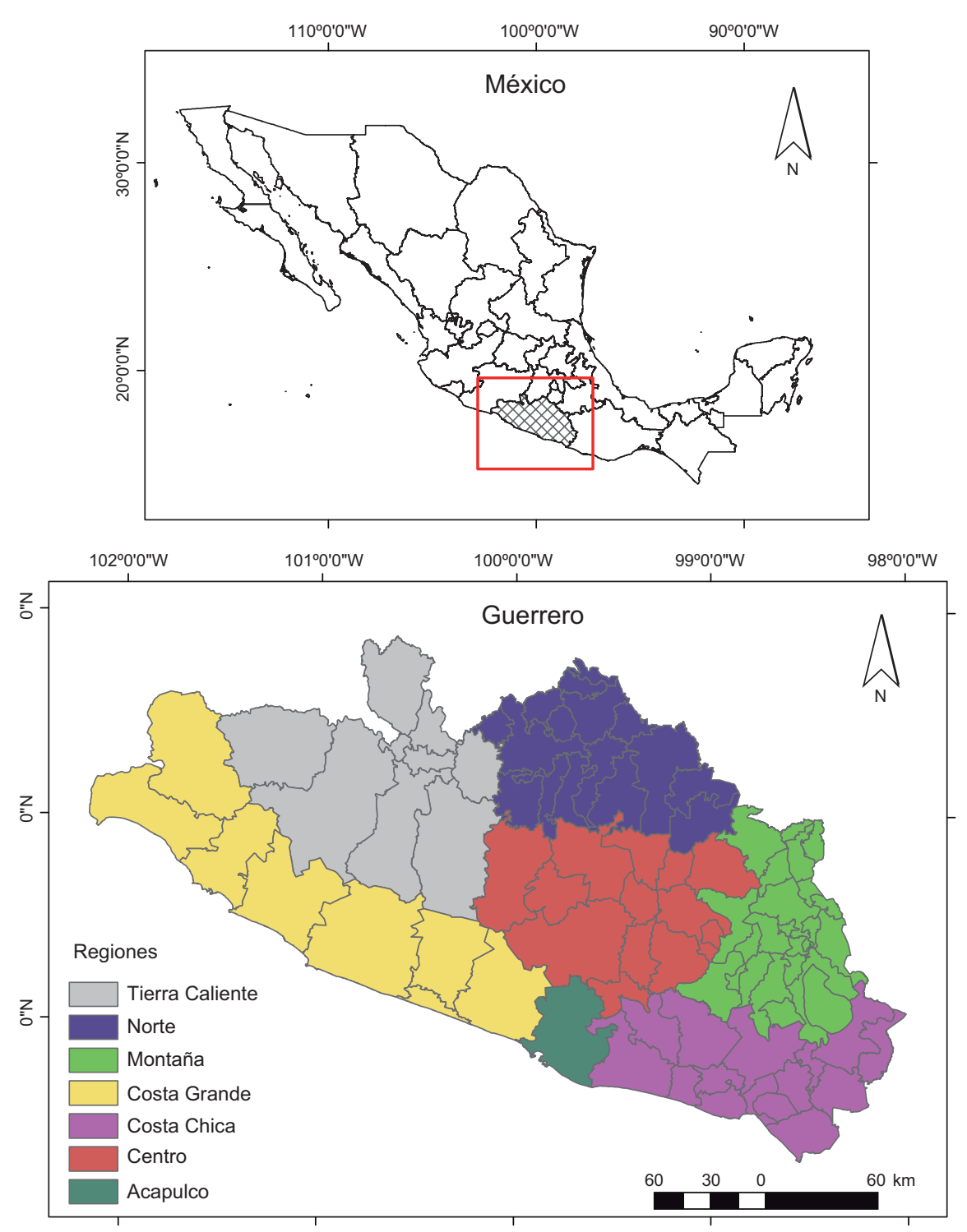

Fig. 1. Localización del estado de Guerrero y su división territorial

durante $7 \mathrm{~min}$, con incremento de $6^{\circ} \mathrm{C}$ cada min hasta alcanzar $250^{\circ} \mathrm{C}$ y continuando a esta temperatura durante 20 min.

Los plaguicidas presentes en la muestra fueron identificados por su tiempo de retención y cuantificados por su área bajo la curva, integrados por el sistema operativo Works Station 6, Varian GC. Se realizaron curvas de calibración con estándares de HCB, isómeros $\alpha-, \beta-$ y $\gamma-\mathrm{HCH}$, pp' y op'DDT y el metabolito pp'diclorodifenildicloroetileno (pp'DDE) (Supelco Inc., Bellefonte, PA, USA). Las unidades en las que se expresaron las concentraciones de plaguicidas organoclorados fueron miligramos por kilogramo de lípidos presentes en la muestra $(\mathrm{mg} / \mathrm{kg})$.

\section{Análisis estadístico}

Las comparaciones de las concentraciones observadas de los plaguicidas en las diferentes regiones del Estado, se llevaron a cabo mediante el uso de la prueba de Kruskal Wallis, de igual forma se compararon los niveles de concentración entre las mujeres procedentes de zonas urbanas y rurales, utilizando las medianas a través de la prueba UMann-Whitney. Todas las pruebas fueron realizadas con un nivel de confianza del $95 \%$ con el programa 
estadístico SPSS, versión 21 (IBM Corporation, Armonk, NY, USA).

\section{Mapas de distribución de la concentración de los plaguicidas}

Los sistemas de información geográfica permiten la interpretación sobre el impacto de ciertos contaminantes en una región específica (Brody et al. 2002). En primera instancia, se realizó un mapa temático en el que se indicaron los municipios de los que procedían las donadoras, para tener una idea clara de la cobertura del muestreo aplicado en este estudio e identificar las jurisdicciones sanitarias visitadas. Posteriormente, el análisis regional se realizó mediante el cálculo de la mediana de cada uno de los contaminantes en las jurisdicciones sanitarias. Las concentraciones fueron expresadas con escalas de colores de modo gradual, es decir, en la manera en que los valores son mayores aumentó la intensidad del color en el mapa. Lo anterior proporcionó una representación gráfica que evidenció de forma rápida y sencilla, la distribución de los plaguicidas en el Estado de Guerrero. Para ello se utilizó el programa ArcGis versión 9.3 (ESRI, Redlands, California, USA).

\section{RESULTADOS Y DISCUSIÓN}

\section{Características de la población de estudio}

Las madres $(\mathrm{n}=171)$ que formaron parte de la población de estudio procedieron de 38 municipios, lo que significa una cobertura de muestreo del $47 \%$, con lo que se logró una adecuada representatividad. Para cada región se obtuvieron los siguientes tamaños de muestra: Tierra Caliente $=23$, Norte $=24$, Centro $=30$, Montaña $=22$, Costa Grande $=23$, Costa Chica $=23$ y Acapulco $=26$. La edad promedio de las donadoras fue de $24.6 \pm 5.9$ años, con un rango entre 14 y 40 años. De cada municipio visitado al menos dos madres fueron seleccionadas para la donación de leche (Fig. 2). Una de las fortalezas de este trabajo es que se obtuvo una cantidad de muestras superior a las recomendaciones de la Organización Mundial de la Salud (OMS) en cuanto al estudio de contaminantes orgánicos persistentes en leche humana (OMS 2007), cuya cifra estipulada es de 50 si se pretende estudiar una población de aproximadamente 50 millones de habitantes. La población de Guerrero es de menos de cuatro millones de personas (INEGI 2010) y las muestras recolectadas fueron más de 170 , por lo que el valor estadístico y las conclusiones que se deriven de los resultados obtenidos son representativos de la región estudiada.

\section{Concentración de plaguicidas organoclorados en las diferentes regiones de Guerrero}

Se detectaron los isómeros pp' y op' DDT y su metabolito pp'DDE. El pp'DDT fue el que mayor concentración presentó respecto al op'DDT (Cuadro I). Esto se debe probablemente a que la formulación del plaguicida DDT que fue utilizado para el control del mosquito transmisor del paludismo estaba constituido en mayor proporción por el pp'DDT ( $85 \%$ ), en tanto que el op'DDT se encontraba en cantidades de $15 \%$ o menos. Los compuestos $\alpha-\mathrm{y} \gamma-\mathrm{HCH}$ no se detectaron en ninguna muestra. De acuerdo con otros estudios, estos isómeros son poco persistentes debido a que su degradación es más rápida tanto en el ambiente como en los sistemas biológicos (Bachmann et al. 1988).

Se observó gran variabilidad en las concentraciones medianas de plaguicidas organoclorados entre las distintas regiones. Las diferencias fueron estadísticamente significativas $(\mathrm{p}<0.05)$ en pp'DDE, op'DDT, pp'DDT y $\Sigma$-DDT (Cuadro II y Fig. 3).

En Tierra Caliente se registraron los niveles más altos del metabolito pp'DDE y los isómeros op'DDT y pp'DDT, con medianas de $1.008,0.027$ y $0.063 \mathrm{mg} / \mathrm{kg}$, respectivamente, mientras que en la región Centro se obtuvieron los valores más bajos de op'DDT $(0.010 \mathrm{mg} / \mathrm{kg})$ y pp'DDT $(0.026 \mathrm{mg} / \mathrm{kg})$. La región de Costa Grande presentó los niveles de pp'DDE más bajos del estado $(0.453 \mathrm{mg} / \mathrm{kg})$, mientras que en la Costa Chica se observaron niveles superiores de este metabolito $(0.885 \mathrm{mg} / \mathrm{kg})$, ligeramente menores que las concentraciones obtenidas en Tierra Caliente $(1.008 \mathrm{mg} / \mathrm{kg})$. Las concentraciones del isómero op'DDT fueron muy similares en todas las regiones estudiadas. De igual forma, el principal isómero del plaguicida DDT, el pp'DDT, presentó valores semejantes en todas las regiones, a excepción de la zona Centro $(0.026 \mathrm{mg} / \mathrm{kg})$ (Cuadro II y Fig. 3). El análisis comparativo de las concentraciones de estos compuestos entre las regiones (Kruskal-Wallis), reveló diferencias estadísticamente significativas ( $\mathrm{p}$ $<0.05)$.

Por su parte, las concentraciones más bajas de $\mathrm{HCB}$ se registraron en la Costa Grande $(0.001 \mathrm{mg} / \mathrm{kg})$ y Tierra Caliente $(0.002 \mathrm{mg} / \mathrm{kg})$, mientras que la más alta se observó en la región Norte con 0.015 $\mathrm{mg} / \mathrm{kg}$. El rango de valores $\beta-\mathrm{HCH}$ abarcó desde $0.002 \mathrm{mg} / \mathrm{kg}$ (Tierra Caliente) hasta $0.018 \mathrm{mg} / \mathrm{kg}$ en la Costa Grande. En ambos casos, las diferencias en las concentraciones de $\mathrm{HCB}$ y $\beta-\mathrm{HCH}$ entre regiones no fueron estadísticamente significativas $(\mathrm{p}>0.05)$ (Cuadro II y Fig. 4). 


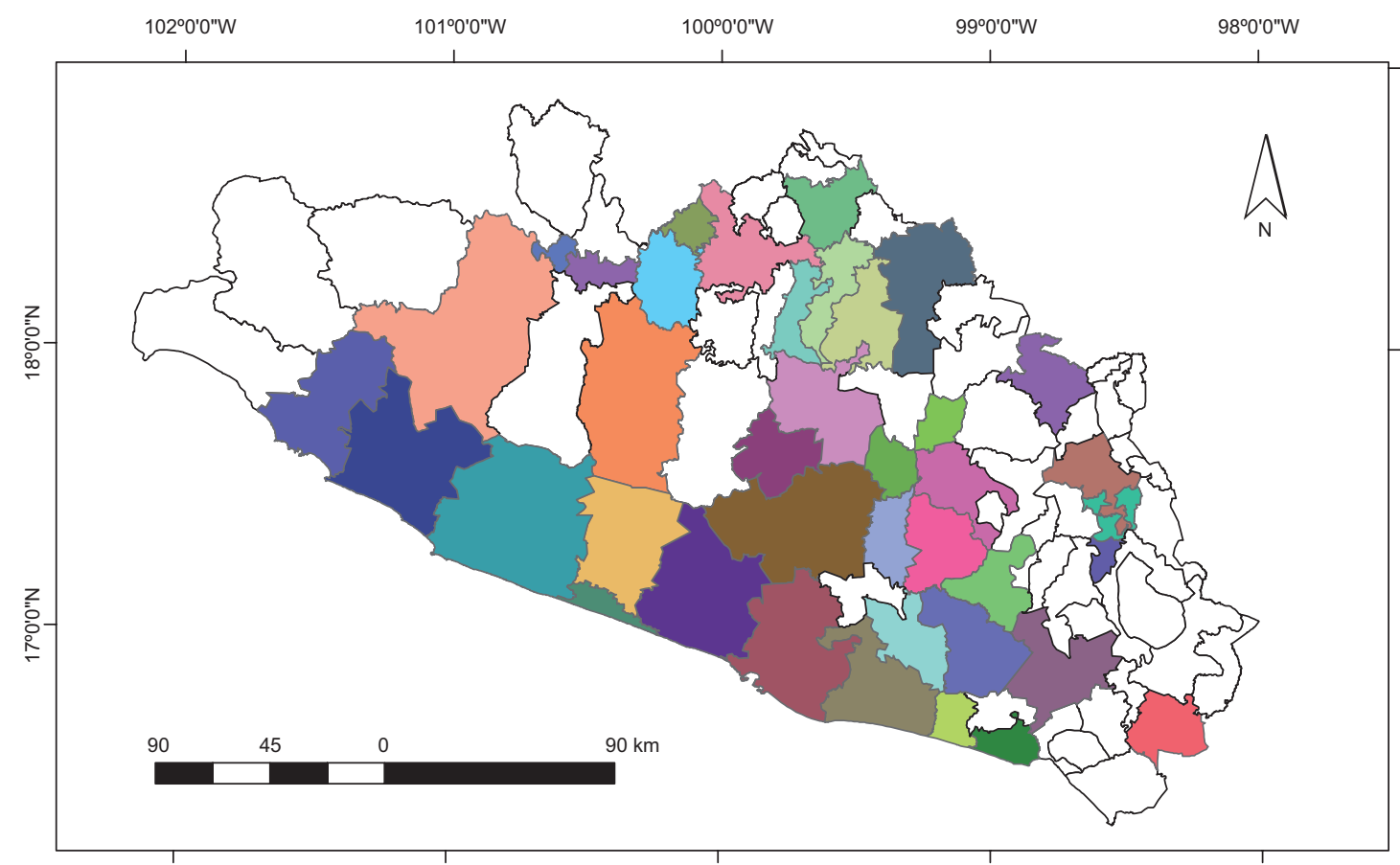

Municipios muestreados

\begin{tabular}{|l|l}
\hline & No muestreados \\
& Acapulco de Juárez \\
\hline & Acatepec \\
\hline & Arcelia \\
\hline Atlamajalcingo del Monte \\
\hline Atoyac de Álvarez \\
\hline Ayutla de los Libres \\
Benito Juárez \\
Chilapa de Ávarez \\
Chilpancingo de los Bravo
\end{tabular}

\begin{tabular}{|l|l}
\hline & Cocula \\
& Copala \\
& Coyuca de Benítez \\
\hline & Coyuca de Catalán \\
\hline & Eduardo Neri \\
\hline & Florencio Villarreal \\
\hline & General Canuto A. Neri \\
\hline & Huitzuco de los Figueroa \\
\hline & Iguala de la Independencia \\
\hline & Leonardo Bravo
\end{tabular}

\begin{tabular}{|l|l}
\hline & Mochitlán \\
\hline & Olinalá \\
\hline & Ometepec \\
Petatlán \\
\hline Pungarabato \\
\hline Quechultenango \\
San Luis Acatlán \\
San Marcos \\
\hline San Miguel Totolapan \\
\hline Taxco de Alarcón
\end{tabular}

\begin{tabular}{|l|l}
\hline & Tecoanapa \\
\hline & Teloloapan \\
\hline & Tepecoacuilco de Trujano \\
\hline & Tixtla de Guerrero \\
\hline & Tlapa de Comonfort \\
& Tlapehuala \\
\hline Técpan de Galeana \\
\hline Xalpatláhuac \\
\hline Zihuatanejo de Azueta \\
\hline Zitlala
\end{tabular}

Fig. 2. Municipios incluidos en el estudio

CUADRO I. CONCENTRACIONES (mg/kg EN BASE LIPÍDICA) DE PLAGUICIDAS ORGANOCLORADOS EN GUERRERO, MÉXICO

\begin{tabular}{lcccccc}
\hline & HCB & $\beta$-HCH & pp'DDE & op'DDT & pp'DDT & $\Sigma$-DDT \\
\hline Frecuencia (\%) & 37 & 36 & 100 & 76 & 98 & - \\
Mediana & 0.009 & 0.004 & 0.760 & 0.016 & 0.045 & 0.833 \\
Rango & $0.001-0.074$ & $0.001-0.123$ & $0.017-5.604$ & $0.001-0.179$ & $0.002-0.727$ & $0.017-5.896$ \\
\hline
\end{tabular}

HCB: hexaclorobenceno; $\beta$-HCH: $\beta$-hexaclorociclohexano; pp'DDE: pp'diclorodifenildicloroetileno; op'DDT: op'diclorodifeniltricloroetano; pp'DDT: pp'diclorodifeniltricloroetano; $\Sigma$-DDT: suma de derivados del diclorodifeniltricloroetano 
CUADRO II. MEDIANAS DE LAS CONCENTRACIONES (mg/kg EN BASE LIPÍDICA) DE PLAGUICIDAS ORGANOCLORADOS DE LAS REGIONES DE GUERRERO

\begin{tabular}{lcccccccc}
\hline & $\begin{array}{c}\text { Tierra } \\
\text { Caliente }\end{array}$ & Norte & Centro & Montaña & $\begin{array}{c}\text { Costa } \\
\text { Grande }\end{array}$ & $\begin{array}{c}\text { Costa } \\
\text { Chica }\end{array}$ & Acapulco & p $^{*}$ \\
\hline HCB & 0.002 & 0.015 & 0.009 & 0.007 & 0.001 & 0.009 & 0.010 & 0.520 \\
$\beta$-HCH & 0.002 & 0.007 & 0.005 & 0.004 & 0.018 & 0.005 & 0.004 & 0.605 \\
pp'DDE & 1.008 & 0.712 & 0.541 & 0.751 & 0.453 & 0.885 & 0.783 & 0.039 \\
op'DDT & 0.027 & 0.017 & 0.010 & 0.021 & 0.018 & 0.016 & 0.010 & 0.001 \\
pp'DDT & 0.063 & 0.048 & 0.026 & 0.049 & 0.043 & 0.060 & 0.048 & 0.001 \\
$\Sigma$-DDT & 1.098 & 0.777 & 0.577 & 0.821 & 0.514 & 0.961 & 0.841 & 0.023 \\
$\Sigma$-plaguicidas & 1.102 & 0.799 & 0.591 & 0.832 & 0.533 & 0.975 & 0.855 & - \\
pp'DDE/ pp'DDT & 16.0 & 14.8 & 20.8 & 15.3 & 10.5 & 14.7 & 16.3 & - \\
\hline
\end{tabular}

*Prueba de Kruskal-Wallis

HCB: hexaclorobenceno; $\beta$-HCH: $\beta$-hexaclorociclohexano; pp'DDE: pp'diclorodifenildicloroetileno; op'DDT: op'diclorodifeniltricloroetano; pp'DDT: pp'diclorodifeniltricloroetano; $\Sigma$-DDT: suma de derivados del diclorodifeniltricloroetano; $\Sigma$-plaguicidas: suma de todos los plaguicidas
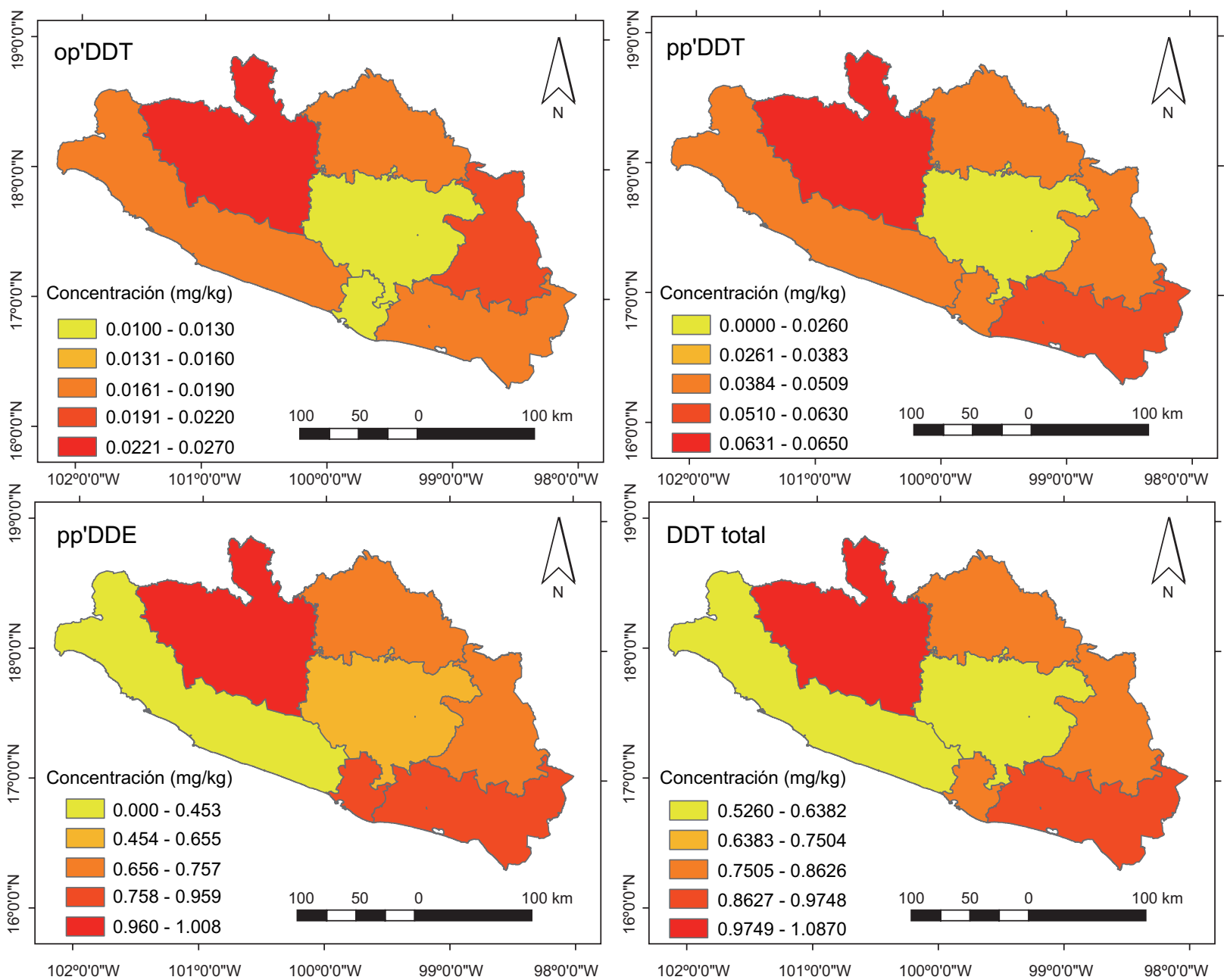

Fig. 3. Distribución espacial de las concentraciones ( $\mathrm{mg} / \mathrm{kg}$ en base lipídica) de DDT's en leche materna en Guerrero. pp'DDE: pp'diclorodifenildicloroetileno; op'DDT: op'diclorodifeniltricloroetano; pp'DDT: pp'diclorodifeniltricloroetano; $\Sigma$-DDT: suma de derivados del diclorodifeniltricloroetano 

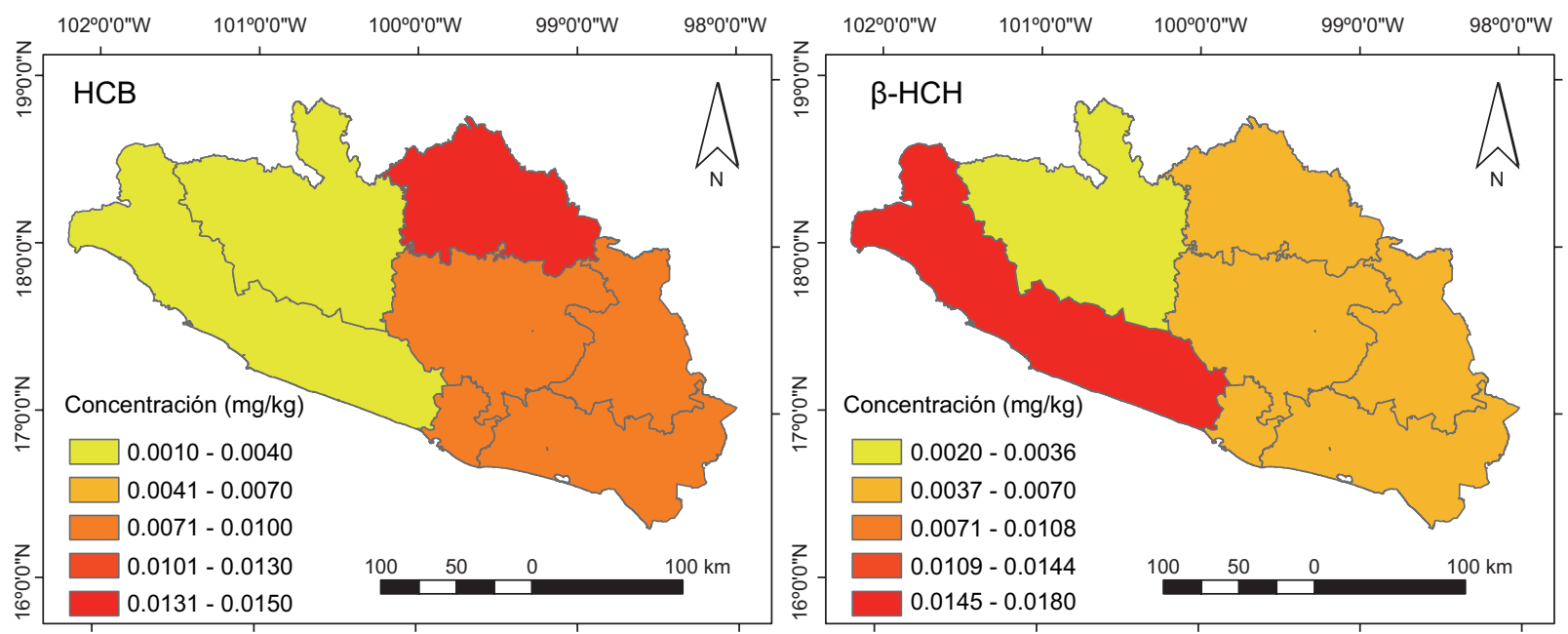

Fig. 4. Distribución espacial de las concentraciones $(\mathrm{mg} / \mathrm{kg}$ en base lipídica) de $\mathrm{HCB}$ y $\beta-\mathrm{HCH}$ en leche materna en Guerrero. HCB: hexaclorobenceno: $\beta$-HCH: $\beta$-hexaclorociclohexano

La suma de las medianas de los plaguicidas muestra que las regiones menos contaminadas del estado fueron la Costa Grande y Centro, con 0.533 y 0.591 $\mathrm{mg} / \mathrm{kg}$, respectivamente, mientras que las regiones con mayor carga de estos contaminantes fueron Tierra Caliente $(1.102 \mathrm{mg} / \mathrm{kg})$ y Costa Chica $(0.975 \mathrm{mg} / \mathrm{kg})$ (Cuadro II). Las proporciones relativas de cada plaguicida en las regiones fueron variables, pero existieron algunos datos que deben resaltarse, como el caso de la región Tierra Caliente que tuvo porcentajes bajos de $\mathrm{HCB}$ y $\beta-\mathrm{HCH}$; por el contrario, el pp'DDE fue el compuesto que predominó entre las habitantes de esta región. También, en el caso de la Costa Chica, el pp'DDT fue el segundo compuesto de importancia con casi un $8 \%$ del total (Fig. 5).

Cabe mencionar que éste es uno de los primeros estudios que se realizan en Guerrero sobre la determinación de plaguicidas organoclorados en leche materna. Por consiguiente, no se tienen puntos de comparación que permitan establecer una discusión con un estado anterior, la tendencia de los niveles de contaminación en el tiempo, y el análisis regional de los segmentos de la población. De ahí la relevancia de este trabajo como guía de referencia enfocada a las concentraciones de plaguicidas en los diferentes estratos de la población, lo cual permitirá la planeación de investigaciones futuras.

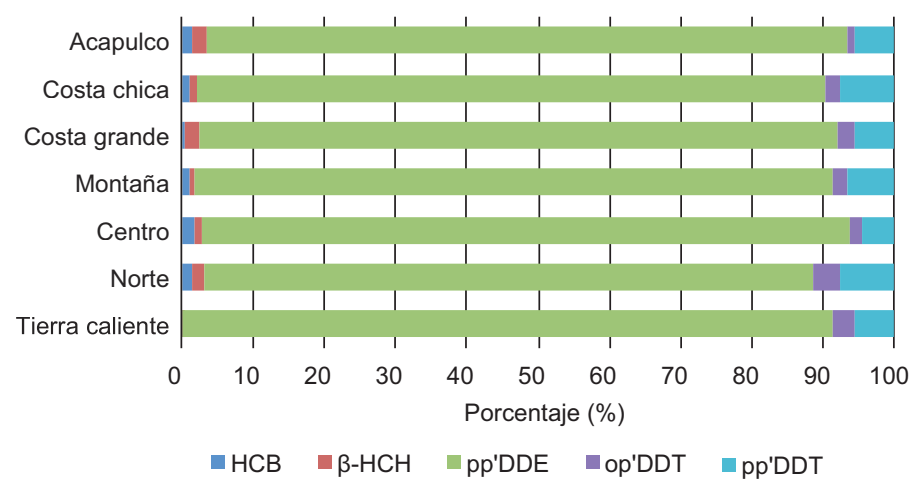

Fig. 5. Proporción relativa (\%) de los plaguicidas entre las regiones del estado de Guerrero. HCB: hexaclorobenceno: $\beta-\mathrm{HCH}$ : $\beta$-hexaclorociclohexano; pp'DDE: pp'diclorodifenildiclor oetileno; op'DDT: op'diclorodifeniltricloroetano; pp'DDT: pp'diclorodifeniltricloroetano 


\section{Plaguicidas organoclorados en zonas rurales y urbanas}

En la comparación de los niveles de plaguicidas organoclorados en leche de mujeres provenientes tanto de localidades urbanas como rurales, los resultados revelan que las concentraciones son prácticamente iguales en los casos del $\beta$-HCH (urbana: $0.005 \mathrm{mg} / \mathrm{kg}$; rural: $0.004 \mathrm{mg} / \mathrm{kg}$ ), op'DDT (urbana: $0.015 \mathrm{mg} / \mathrm{kg}$; rural: $0.017 \mathrm{mg} / \mathrm{kg}$ ) y pp'DDT (urbana: $0.045 \mathrm{mg} / \mathrm{kg}$; rural: $0.049 \mathrm{mg} / \mathrm{kg}$ ). Las concentraciones más altas de pp'DDE se presentaron en las zonas rurales $(1.008 \mathrm{mg} /$ $\mathrm{kg}$ ), mientras que en zonas urbanas fueron inferiores $(0.747 \mathrm{mg} / \mathrm{kg})$. No obstante, la diferencia entre ambas medianas no fue estadísticamente significativa $(\mathrm{p}>0.05)$ (Fig. 6). La misma situación se observó respecto del DDT total: en localidades urbanas se obtuvieron medianas de $0.826 \mathrm{mg} / \mathrm{kg}$ y en las rurales de $1.079 \mathrm{mg} / \mathrm{kg}$, que fueron estadísticamente iguales $(\mathrm{p}>0.05)$. El caso contrario se presentó con el HCB, cuya mediana fue de $0.009 \mathrm{mg} / \mathrm{kg}$ en localidades urbanas y de $0.015 \mathrm{mg} / \mathrm{kg}$ en localidades rurales; la diferencia fue significativa $(\mathrm{p}<0.05)$ (Fig. 6). Los datos presentados indican que las
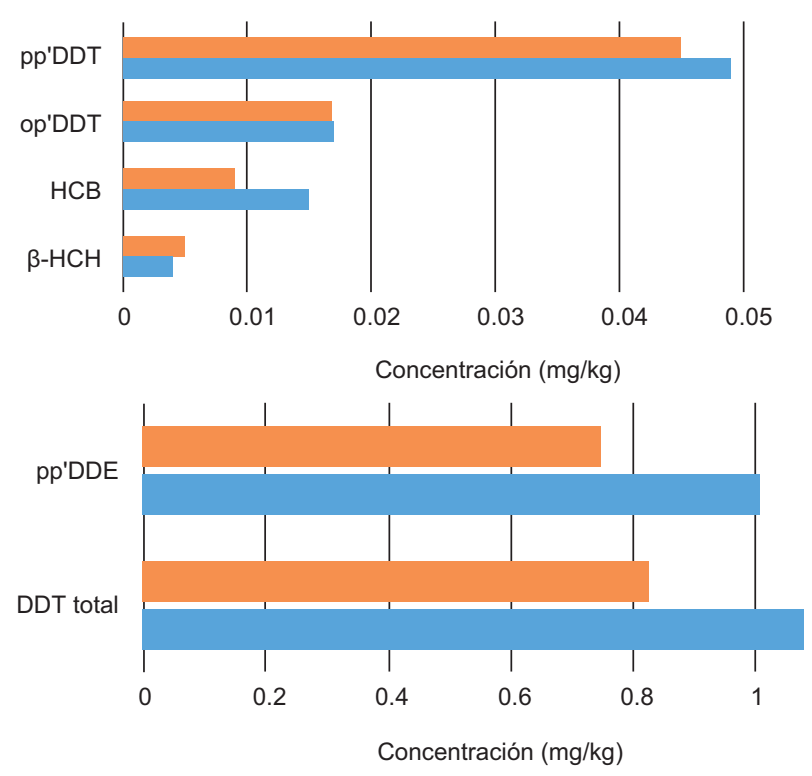

$$
\text { Urbana Rura }
$$

Fig. 6. Concentraciones ( $\mathrm{mg} / \mathrm{kg}$ en base lipídica) de plaguicidas en localidades rurales y urbanas. HCB: hexaclorobenceno: $\beta$-HCH: $\beta$-hexaclorociclohexano; pp'DDE: pp'diclorodifenildicloroetileno; op'DDT: op'diclorodifeniltricloroetano; pp'DDT: pp'diclorodifeniltricloroetano; $\Sigma$-DDT: suma de derivados del diclorodifeniltricloroetano habitantes de las zonas rurales tienen un grado mayor de exposición a los plaguicidas estudiados que las mujeres que habitan en localidades urbanas, lo cual se explica por el hecho de que la aplicación de plaguicidas fue más intensa como consecuencia de las actividades sanitarias llevadas a cabo en el medio rural. Los resultados de la suma de las medianas de cada uno de estos compuestos fueron superiores en habitantes de zonas rurales $(1.312 \mathrm{mg} / \mathrm{kg})$ que en zonas urbanas $(0.899 \mathrm{mg} / \mathrm{kg})$, lo que es un reflejo de la contaminación ambiental originada por los compuestos organoclorados presentes en estos lugares.

Esta situación es preocupante, si se tiene en cuenta que en Guerrero 1416505 habitantes se ubican en zonas rurales, lo que representa el $41.8 \%$ de la población total (INEGI 2010). Por lo tanto, un amplio sector de la población guerrerense reside en sitios que favorecen el contacto con contaminantes ambientales persistentes como los plaguicidas organoclorados, lo cual se refleja en el grado de contaminación de la leche que presentan las mujeres de dichas zonas.

Estos resultados son consistentes con los reportados por Waliszewski et al. (1996), que encontraron en leche de madres de zonas suburbanas de Veracruz, México, concentraciones mayores de $\Sigma-\mathrm{HCH}$ $(0.806 \mathrm{mg} / \mathrm{kg}), \mathrm{pp}$ 'DDE $(8.253 \mathrm{mg} / \mathrm{kg})$ y pp'DDT $(2.460 \mathrm{mg} / \mathrm{kg})$ que en las de zonas urbanas $(\Sigma-\mathrm{HCH}$ $=0.387, \mathrm{pp}$ 'DDE $=2.709$ y pp'DDT $=0.422 \mathrm{mg} / \mathrm{kg}$ ). En un estudio realizado por Prado et al. (2004) en la Ciudad de México, se obtuvieron valores similares de $\gamma-\mathrm{HCH}$ en zonas rurales $(0.300 \mathrm{mg} / \mathrm{kg})$ y en zonas urbanas $(0.260 \mathrm{mg} / \mathrm{kg})$. En Bélgica, Colles et al. (2008) analizaron muestras de leche de habitantes con un considerable componente rural (Valonia) y localidades netamente urbanas como Flandes y Bruselas. La primera región presentó niveles de $\mathrm{HCB}=0.017 \mathrm{mg} / \mathrm{kg}$ y pp'DDE $=$ $0.085 \mathrm{mg} / \mathrm{kg}$, mientras que en Flandes el HCB fue de $0.016 \mathrm{mg} / \mathrm{kg}$ y el pp'DDE de $0.118 \mathrm{mg} / \mathrm{kg}$. En Bruselas se encontraron concentraciones parecidas $(\mathrm{HCB}=0.017$ y pp'DDE $=0.090 \mathrm{mg} / \mathrm{kg})$. Por último, en Túnez las concentraciones de DDT total de mujeres de zonas rurales tuvieron valores superiores $(0.059 \mathrm{mg} / \mathrm{kg})$ en comparación con las que habitan en zonas urbanas $(0.018 \mathrm{mg} / \mathrm{kg}$ ) (Ennaceur et al. 2008).

Finalmente, las proporciones relativas de estos compuestos orgánicos persistentes en las habitantes de ambas zonas en el estado de Guerrero mostraron patrones de distribución muy parecidos o prácticamente idénticos, con predominancia del pp'DDE y 
un reducido porcentaje del pp'DDT (Fig. 7). Los demás compuestos tuvieron algunas diferencias, pero no fueron estadísticamente significativas.

Los resultados revelan la magnitud de la contaminación ambiental por plaguicidas organoclorados y proporcionan una base fundamental con relación a sus posibles implicaciones en la salud de quienes habitan en las diferentes regiones de Guerrero. Esta información también es de utilidad para que las instituciones encargadas del cuidado de la salud y el ambiente puedan planear y ejecutar acciones de intervención en las zonas contaminadas, con el fin de disminuir y/o eliminar la posibilidad de que los habitantes continúen expuestos a estos plaguicidas y de esta forma erradicar su presencia en el organismo.
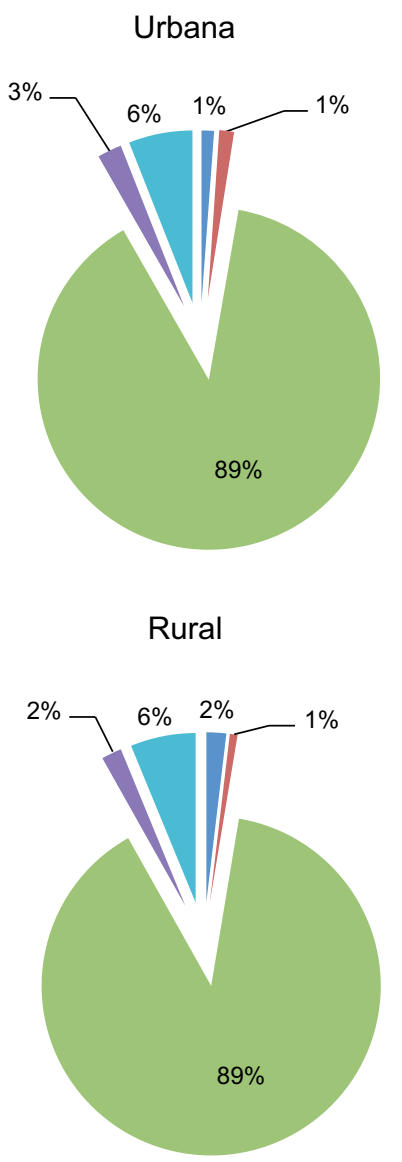

口CB $\quad$ B-HCH $\quad$ pp'DDE $\quad$ op'DDT

Fig. 7. Proporción relativa del impacto de los plaguicidas en el área urbana y rural del Estado de Guerrero. HCB: hexaclorobenceno: $\beta$-HCH: $\beta$-hexaclorociclohexano; pp'DDE: pp'diclorodifenildicloroetileno; op'DDT: op'diclorodifeniltricloroetano; pp'DDT: pp'diclorodifeniltricloroetano

\section{CONCLUSIONES}

Se encontraron residuos de plaguicidas organoclorados como el HCB, $\beta$-HCH, pp'DDE, op'DDT y pp'DDT en la mayoría de las muestras de leche materna de la población estudiada. A pesar de que estos compuestos fueron aplicados en décadas pasadas, aún persisten en el ambiente, especialmente en el suelo superficial. Como resultado, continúa la exposición de los seres humanos, que se refleja en su detección en la leche de las madres que formaron parte de este estudio.

Se observó una presencia diferenciada de estos contaminantes en las regiones que conforman el estado de Guerrero, ya que los compuestos derivados del DDT presentaron mayores concentraciones en la Tierra Caliente y la Costa Chica, en tanto que las regiones Norte y Costa Grande exhibieron concentraciones superiores de $\mathrm{HCB}$ y $\beta-\mathrm{HCH}$, respectivamente. También se detectó mayor exposición en las mujeres procedentes de localidades rurales, puesto que tuvieron niveles más altos de plaguicidas respecto a las que habitan en el medio urbano. Esto tiene implicaciones importantes, ya que el territorio guerrerense posee un fuerte componente rural; por lo tanto, un segmento importante de la población se encuentra expuesto a estos contaminantes.

Por último, este trabajo demostró el grado de contaminación existente en el territorio de Guerrero como resultado de la aplicación de estos compuestos orgánicos persistentes en el pasado, y aporta información relevante a la comunidad científica que puede aprovecharse en investigaciones futuras.

\section{AGRADECIMIENTOS}

Este trabajo fue financiado por el Fondo Mixto del Consejo Nacional de Ciencia y Tecnología-Gobierno del Estado de Guerrero, proyecto con clave GUE2009-01-126320. Nuestra gratitud para todas las mujeres que desinteresadamente proporcionaron una muestra para hacer posible este estudio.

\section{REFERENCIAS}

Bachmann A., Walet P., Wijnen P., De Bruin W., Huntjens J.L.M., Roelofsen W. y Zehnder A.J.B. (1988). Biodegradation of alpha- and beta-hexachlorocyclohexane in a soil slurry under different redox conditions. Appl. Environ. Microbiol. 54 (1), 143-149. 
Brody J.G., Vorhees D.J., Melly S.J., Swedis S.R., Drivas P.J. y Rudel R.A. (2002). Using GIS and historical records to reconstruct residential exposure to large-scale pesticide application. J. Expo. Sci. Environ. Epidemiol. 12 (1), 64-80. DOI: $10.1038 / \mathrm{sj} /$ jea/7500205

Chávez-Almazán L.A., Díaz-Ortiz J., Alarcón-Romero M., Dávila-Vázquez G., Saldarriaga-Noreña H. y Waliszewski S.M. (2014). Organochlorine pesticide levels in breast milk in Guerrero, Mexico. Bull. Environ. Contam. Toxicol. 93 (3), 294-298.

DOI: $10.1007 / \mathrm{s} 00128-014-1308-4$

Chávez-Almazán L.A., Díaz-Ortiz J., Alarcón-Romero M., Dávila-Vázquez G., Saldarriaga-Noreña H., Sampedro-Rosas L., López-Silva S., Santiago-Moreno A., Rosas-Acevedo J.L. y Waliszewski S.M. (2016). Influence of breastfeeding time on levels of organochlorine pesticides in human milk of a Mexican population. Bull. Environ. Contam. Toxicol. 96 (2), 168-172. DOI: $10.1007 / \mathrm{s} 00128-015-1702-6$

Colles A., Koppen G., Hanot V., Nelen V., Noël E., Malisch R., Kotz A., Kypke K., Biot P., Vinkx C. y Schoeters G. (2008). Fourth WHO-coordinated survey of human milk for persistent organic pollutants (POPs): Belgian results. Chemosphere 73 (6), 907-914.

DOI: 10.1016/j.chemosphere.2008.07.002

De Roos A.J., Hartge P., Lubin J.H., Colt J.S., Davis S., Cerhan J.R., Severson R.K., Cozen W., Patterson D.G., Needham L.L. y Rothman N. (2005). Persistent organochlorine chemicals in plasma and risk of nonHodgkin's lymphoma. Cancer Res. 65 (23), 1121411226. DOI:10.1158/0008-5472.CAN-05-1755

Ennaceur S., Gandoura N. y Driss M.R. (2008). Distribution of polychlorinated biphenyls and organochlorine pesticides in human breast milk from various locations in Tunisia: Levels of contamination, influencing factors, and infant risk assessment. Environ. Res. 108 (1), 86-93. DOI: 10.1016/j.envres.2008.05.005

Gallardo-Díaz E.G., Borja-Aburto V.H., Méndez-Galván J.F., Sánchez-Tejeda G., Olguín-Bernal H. y RamírezHernández J.A. (2000). Situación actual de la malaria y el uso del DDT en México [en línea]. http://www.bvsde.paho.org/bvsdepl/fulltext/ddt/MEX.pdf 13/01/2017
INEGI (2010). Censo de población y vivienda 2010. Principales resultados por localidad. Instituto Nacional de Estadística y Geografía [en línea]. http://www.inegi. org.mx/sistemas/consulta_resultados/iter2010.aspx $03 / 01 / 2017$

IPCS (2011). DDT in indoor residual spraying: human health aspects. International Programme on Chemical Safety [en línea]. http://www.who.int/ipcs/publications/ehc/ehc241.pdf 15/08/2016

OMS (2007). Cuarto estudio coordinado por la OMS sobre contaminantes orgánicos persistentes en la leche materna, en colaboración con el Programa de las Naciones Unidas para el Medio Ambiente (PNUMA). Organización Mundial de la Salud [en línea]. http:// www.who.int/foodsafety/chem/POPprotocol_sp.pdf 20/12/2016

Prado G., Díaz G., Noa M., Méndez I., Cisneros I. y Castorena F. (2004). Niveles de pesticidas organoclorados en leche humana de la Ciudad de México. Agro. Sur 32 (2), 60-69.

Raaschou-Nielsen O., Pavuk M., LeBlanc A., Dumas P., Weber J.P., Olsen A., Tjonneland A., Overvad K. y Olsen J.H. (2005). Adipose organochlorine concentrations and risk of breast cancer among postmenopausal Danish women. Cancer Epidemiol. Biomarkers Prev. 14 (1), 67-74.

Waliszewski S.M., Pardio J.N., Chantiri V.T., Infanzón R.M. y Rivera J. (1996). Organochlorine pesticide residues in human breast milk from tropical areas in Mexico. Bull. Environ. Contam. Toxicol. 57 (1), 22-28.

Waliszewski S.M., Gómez-Arroyo S., Carbajal O., Villalobos-Pietrini R. e Infanzón R.M. (2004). Uso del ácido sulfúrico en las determinaciones de plaguicidas organoclorados. Rev. Int. Contam. Ambie. 20 (4), 185-192. 